\title{
Checklist improves communication between doctors and patients and results in changes in care
}

van Os J, Altamura AC, Bobes J, et al. Evaluation of the Two-Way Communication Checklist as a clinical intervention. Results of a multinational, randomised controlled trial. Br J Psychiatry 2004;184:79-83.

\section{Does a checklist intervention help people with schizophrenia express their needs and change their care?}

\section{METHODS}

Design: Randomised controlled trial.
Allocation: Concealed.
Sollow up period: Four to six weeks.
timeframe not stated.
Patients: 134 people (mean age 41 years, $45 \%$ female), with a
DSM-IV diagnosis of schizophrenia, schizoaffective disorder, or
schizophreniform disorder, age ₹18, who were in a stable phase
of illness and in regular outpatient contact with their psychiatric
care team. $20 \%$ of participants were employed and $22 \%$ married.
Exclusion criteria: current or recent inpatient, or those likely to be
admitted to hospital in the near future.
Intervention: Usual care; the Two-Way Communication Checklist
(2-COM): a list of 20 common problems or areas of perceived
needs that might be experienced by people with severe mental
illness - for example, housing, relationships, money, symptoms,
treatment side effects. The checklist prolonged the patient visit by
an average of 13 minutes.
Outcomes: Quality of patient-clinician communication, reported
by the patient; change in clinician behaviour or management,
reported by the clinician.
Patient follow up: Not reported.

\section{MAIN RESULTS}

As reported by patients, 2-COM improved the quality of communication between patient and doctor compared with usual care $(\mathrm{p}=0.031)$. Treatment change was more likely with 2-COM compared with usual care as reported by the doctor $(\mathrm{OR}=3.7$, $\mathrm{p}=0.009, \mathrm{NNT}=6$ ). Those with more reported needs were more likely to have changes in their management. There was no relation between changes in management and severity of illness. Also, the needs most likely to induce treatment change were those unrelated to medication.

\section{CONCLUSIONS}

The quality of patient-doctor communication and changes in management can be enhanced by discussion of patient needs using an established checklist which has been completed by the patient. Simple, non-pharmacological interventions may be explored in this way.

For correspondence: Jim van Os, Department of Psychiatry and Neuropsychiatry, South Limburg Mental Health Research Network, Maastricht University, Maastricht, the Netherlands; j.vanos@sp.unimaas.nl Sources of funding: This study was funded by an unrestricted grant from Astra Zeneca.
Commentary

This paper is widely relevant as it addresses a phenomenon at the heart of clinical practice: professional-patient communication.

Although patients regard the quality of the therapeutic relationship as the most crucial factor in good psychiatric care, ${ }^{1}$ and a better professional-patient relationship predicts better patient outcome, ${ }^{2-4}$ specific research on how to improve communication has been lacking.

This study is timely in redressing this gap and in showing how a brief communication checklist can improve professional-patient communication and clinical management. As the authors note, it is tempting to speculate that such an improvement in communication and clinical management might, in turn, have a positive effect on long term patient outcome. Two intervention studies with a similar focus on patients' needs for care, which assess long term outcome, are currently underway: the European MECCA study, 6 and the UK FOCUS study. Both aim to assess the impact of routinely assessing patients' views on their needs for care and quality of life on the therapeutic relationship and patient outcome over a 12 and 18 month period respectively.

This intervention could be implemented in most mental health treatment contexts as long as the patient is able and willing to complete the checklist. It is encouraging that (1) the checklist was well regarded by patients, (2) severity of illness was not a barrier to using the checklist in the consultation and subsequent treatment change, and (3) patients with more needs and greater impairment benefited most.

However, an obstacle for clinicians will be that it lengthened the visit by 13 minutes on average. This is longer than some routine outpatient consultations (for example, in busy urban services in the UK). To increase acceptability among clinicians, the next challenge will be to identify if (and how) facilitating patients in focussing on their needs can become standard practice in service encounters without significantly increasing their length. Research which analyses how professionals and patients actually communicate in the consultation itself, ${ }^{9} 9$ to discriminate more and less effective patterns of communicative engagement, may offer a way forward.

Rosemarie McCabe, PhD

Barts and the London School of Medicine, London, UK

1 Johansson H, Eklund M. Patients' opinion on what constitutes good psychiatric care. Scand J Caring Sci 2003;17:339-46.

2 Cruz M, Pincus HA. Research on the influence that communication in psychiatric encounters has on treatment. Psychiatr Serv 2002;53:1253-65.

3 Weiss M, Gaston L, Propst A, ef al. The role of the alliance in the

pharmalogic treatment of depression. J Clin Psychiatry 1997:58:196-204.

4 McCabe R, Priebe S. The therapeutic relationship in the treatment of severe mental illness: A review of methods and findings. Int J Soc Psychiatry (in press).

5 McCabe R, Priebe S. Focussing on quality of life in treatment. Int Rev Psychiatry 2002;14:225-30

6 Priebe S, Bullenkamp J, McCabe R, et al. The impact of routine outcome measurement on treatment processes in community mental health care: Approach and methods of the MECCA study. Epidem Psichiatr Soc 2002:11:198-205.

7 Slade $M$. What outcomes to measure in routine mental health services and how to assess them: A systematic review. Aust NZ I Psychiatry 2002; 36:743-53

8 Drew P, Chatwin J, Collins S. Conversation analysis: A method for research into interactions between patients and health-care professionals. Health Expect 2001;4:58-70.

9 McCabe R, Priebe S, Burns T, et al. Engagement of patients with psychosis in the consultation: $A$ conversation analytic study. BMJ $2002 ; 325: 1148-51$ 